\title{
Yang-Baxter deformations and string dualities
}

\author{
Takuya Matsumoto $^{a}$ and Kentaroh Yoshida ${ }^{b}$ \\ ${ }^{a}$ Institute for Advanced Research and Department of Mathematics, Nagoya University, \\ Nagoya 464-8602, Japan \\ ${ }^{b}$ Department of Physics, Kyoto University, \\ Kyoto 606-8502, Japan \\ E-mail: takuya.matsumoto@math.nagoya-u.ac.jp, \\ kyoshida@gauge.scphys.kyoto-u.ac.jp
}

ABSTRACT: We further study integrable deformations of the $\mathrm{AdS}_{5} \times \mathrm{S}^{5}$ superstring by following the Yang-Baxter sigma model approach with classical $r$-matrices satisfying the classical Yang-Baxter equation (CYBE). Deformed string backgrounds specified by $r$-matrices are considered as solutions of type IIB supergravity, and therefore the relation between gravitational solutions and $r$-matrices may be called the gravity/CYBE correspondence. In this paper, we present a family of string backgrounds associated with a classical $r$-matrices carrying two parameters and its three-parameter generalization. The two-parameter case leads to the metric and NS-NS two-form of a solution found by Hubeny-Rangamani-Ross [hep-th/0504034] and another solution in [arXiv:1402.6147]. For all of the backgrounds associated with the three-parameter case, the metric and NS-NS two-form are reproduced by performing TsT transformations and S-dualities for the undeformed $\mathrm{AdS}_{5} \times \mathrm{S}^{5}$ background. As a result, one can anticipate the R-R sector that should be reproduced via a supercoset construction.

Keywords: AdS-CFT Correspondence, Integrable Field Theories, Sigma Models

ARXIV EPRINT: 1412.3658 


\section{Contents}

1 Introduction 1

2 Jordanian deformations of the $\mathrm{AdS}_{5} \times \mathrm{S}^{5}$ superstring 3

2.1 Yang-Baxter deformations of the $\mathrm{AdS}_{5} \times \mathrm{S}^{5}$ superstring 3

2.2 A two-parameter deformation of $\mathrm{AdS}_{5}$

2.3 The metric and NS-NS two-form 5

3 A chain of dualities for $\mathrm{AdS}_{5} \times \mathrm{S}^{5} \quad 6$

$\begin{array}{ll}3.1 \text { The first TsT transformation } & 7\end{array}$

3.2 The second TsT transformation 8

$\begin{array}{ll}3.3 \text { S-duality } & 9\end{array}$

3.4 The third TsT transformation 9

4 Duality-chains and classical $r$-matrices $\quad 10$

$\begin{array}{ll}4.1 \text { The fourth TsT transformation } & 11\end{array}$

$\begin{array}{ll}4.2 \text { The second S-duality } & 11\end{array}$

$\begin{array}{lll}4.3 & \text { A three-parameter deformation of } \mathrm{AdS}_{\mathbf{5}} \times \mathrm{S}^{\mathbf{5}} & 12\end{array}$

5 Conclusion and discussion $\quad 13$

$\begin{array}{lr}\text { A Notation and convention } & 14\end{array}$

$\begin{array}{ll}\text { B The T-duality rules } & 15\end{array}$

\section{Introduction}

The AdS/CFT correspondence [1] has a significant property, integrability [2]. It enables us to compute exactly some quantities such as scaling dimensions of composite operators and scattering amplitudes at arbitrary couplings even though those are not protected by supersymmetries. On the string-theory side, the integrability has an intimate relation to classical integrability of non-linear sigma models in two dimensions. In particular, the mathematical structure of the target-space geometry is closely associated with the integrability.

The supergeometry of $\mathrm{AdS}_{5} \times \mathrm{S}^{5}$ is represented by the following supercoset,

$$
\frac{\operatorname{PSU}(2,2 \mid 4)}{\mathrm{SO}(1,4) \times \mathrm{SO}(5)}
$$

The Green-Schwarz type action is constructed based on this supercoset [3]. It is known that the coset (1.1) enjoys the $\mathbb{Z}_{4}$-grading property and it ensures the classical integrability [4]. For another formulation [5], the classical integrability is discussed in [6, 7]. 
What kinds of string backgrounds would be concerned with integrability? It is wellknown that symmetric cosets in general lead to classical integrability. For the symmetric cosets, consistent string backgrounds have been classified in [8, 9]. On the other hand, some of non-symmetric cosets may lead to integrable sigma models, but there is no general prescription. Thus one has to study case by case and hence the main focus has been on simple cases. In the case of squashed $\mathrm{S}^{3}$, a $q$-deformation of $\mathfrak{s u}(2)$ and its affine extension have been shown in [10-14]. Similar results have also been obtained for 3D Schrödinger spacetimes [15-18]. For an earlier attempt to study higher-dimensional cases, see [19]. For integrable deformations of Wess-Zumino-Novikov-Witten models, see [20-26].

A systematic method to study integrable deformations was proposed by Klimcik [2730]. The original form was applicable only to principal chiral models and it was based on classical $r$-matrices satisfying modified classical Yang-Baxter equation (mCYBE). Then it was generalized to symmetric coset cases [31]. Just after that, it was further applied to a $q$-deformation of the $\operatorname{AdS}_{5} \times \mathrm{S}^{5}$ superstring [32, 33]. The $q$-deformed metric (in the string frame) and the NS-NS two-form were derived in [34]. Notably, the metric exhibits a singularity surface. A generalization to other cases are studied in [35]. A mirror description has been proposed in $[36,37]$. A possible resolution of the singularity has been argued by taking the fast-moving string limit $[38,39]$. Giant magnon solutions are studied in [36, 4042]. Deformed Neumann models are constructed [43]. A new coordinate system has been argued in $[44,45]$. Although it seems quite difficult to fix the dilaton and the R-R sector for the $q$-deformed $\mathrm{AdS}_{5} \times \mathrm{S}^{5}$ superstring, the dilaton was determined at least in lowerdimensional cases such as $\mathrm{AdS}_{2} \times \mathrm{S}^{2}$ [46]. For two-parameter generalizations, see [35, 47].

It is also possible to consider another kind of integrable deformations of the $\operatorname{AdS}_{5} \times \mathrm{S}^{5}$ superstring based on the classical Yang-Baxter equation (CYBE) [48], rather than mCYBE. We have already found out some classical $r$-matrices, which correspond to solutions of type IIB supergravity. An example is the Lunin-Maldacena-Frolov backgrounds [49, 50] and the corresponding $r$-matrix is composed of the Cartan generators of $\mathfrak{s u}(4)$ [51]. Another example is gravity duals for non-commutative gauge theories [52-55]. The associated $r$ matrices are of peculiar Jordanian type [56]. A rather remarkable example is the $\operatorname{AdS}_{5} \times T^{1,1}$ case. The $T^{1,1}$ background is argued to be non-integrable [57]. On the other hand, the $T^{1,1}$ geometry can be represented by a coset and hence one may consider Yang-Baxter deformations of $T^{1,1}$. Then it has been shown in [58] that the resulting geometry nicely agrees with $\gamma$-deformations of $T^{1,1}$ previously discussed in [49, 59]. For a short summary of the works based on the CYBE, see [60].

In this paper, we further study integrable deformations of the $\mathrm{AdS}_{5} \times \mathrm{S}^{5}$ superstring along the line of [48]. Deformed string backgrounds specified by $r$-matrices are considered as solutions of type IIB supergravity, and therefore the relation between gravitational solutions and $r$-matrices may be called the gravity/CYBE correspondence. We will present here a family of string backgrounds associated with a classical $r$-matrices carrying two parameters and its three-parameter generalization. The two-parameter case leads to the metric and NS-NS B-field of a solution found by Hubeny-Rangamani-Ross [61] and another solution in [62] as special cases. More generally, for all of the backgrounds associated with the three-parameter case, the metric and NS-NS two-form are reproduced by performing TsT 
transformations and S-dualities for the undeformed $\mathrm{AdS}_{5} \times \mathrm{S}^{5}$ background. As a result, one can anticipate the R-R sector that should be reproduced via a supercoset construction.

A remarkable point is that the solution obtained in [62] has been reproduced by performing a duality chain for the $\mathrm{AdS}_{5} \times \mathrm{S}^{5}$, and as a result it is shown to be a consistent string background. Namely, it is automatically ensured that the beta function vanishes. This point should be stressed against those who are skeptical about the solution in [62].

This paper is organized as follows. Section 2 provides a short review of Yang-Baxter deformations of the $\mathrm{AdS}_{5} \times \mathrm{S}^{5}$ superstring. We present a classical $r$-matrix with two parameters, which is a solution of the CYBE. Then the deformed metric and NS-NS two-form are obtained by evaluating the classical action. In section 3 , we reproduce the resulting metric and NS-NS two-form by performing a chain of dualities for the $\mathrm{AdS}_{5} \times \mathrm{S}^{5}$ background. Section 4 generalizes the previous argument to a three-parameter case and considers the correspondence between the deformations of $\mathrm{AdS}_{5} \times \mathrm{S}^{5}$ and the associated classical $r$-matrices. Section 5 is devoted to conclusion and discussion.

In appendix A, our notation and convention are summarized. The Buscher rules of T-duality are listed in appendix B.

\section{Jordanian deformations of the $\mathrm{AdS}_{5} \times \mathrm{S}^{5}$ superstring}

In subsection 2.1, let us first recall the formulation of Yang-Baxter sigma models. Then we consider a two-parameter deformation of the $\mathrm{AdS}_{5}$ in subsection 2.2. The resulting metric and NS-NS two-form are computed in subsection 2.3.

\subsection{Yang-Baxter deformations of the $\operatorname{AdS}_{5} \times \mathrm{S}^{5}$ superstring}

The Yang-Baxter sigma model approach [27-33] is applicable to the $\mathrm{AdS}_{5} \times \mathrm{S}^{5}$ superstring by using a classical $r$-matrix satisfying the CYBE [48]. Then the deformed action is given by

$$
S=-\frac{1}{4}\left(\gamma^{\alpha \beta}-\epsilon^{\alpha \beta}\right) \int_{-\infty}^{\infty} d \tau \int_{0}^{2 \pi} d \sigma \operatorname{STr}\left(A_{\alpha} d \circ \frac{1}{1-\eta R_{g} \circ d} A_{\beta}\right)
$$

where the left-invariant one-form is defined as

$$
A_{\alpha} \equiv g^{-1} \partial_{\alpha} g, \quad g \in \mathrm{SU}(2,2 \mid 4) .
$$

By taking the parameter $\eta \rightarrow 0$, the action (2.1) reduces to the undeformed one [3]. Here the flat metric $\gamma^{\alpha \beta}$ and the anti-symmetric tensor $\epsilon^{\alpha \beta}$ on the string world-sheet are normalized as $\gamma^{\alpha \beta}=\operatorname{diag}(-1,1)$ and $\epsilon^{\tau \sigma}=1$, respectively. The operator $R_{g}$ is defined as

$$
R_{g}(X) \equiv g^{-1} R\left(g X g^{-1}\right) g,
$$

where a linear operator $R$ is a solution of CYBE rather than mCYBE. The R-operator is related to a classical $r$-matrix in the tensorial notation through

$$
\begin{aligned}
R(X) & =\operatorname{STr}_{2}[r(1 \otimes X)]=\sum_{i}\left(a_{i} \operatorname{STr}\left(b_{i} X\right)-b_{i} \operatorname{STr}\left(a_{i} X\right)\right) \\
\text { with } \quad r & =\sum_{i} a_{i} \wedge b_{i} \equiv \sum_{i}\left(a_{i} \otimes b_{i}-b_{i} \otimes a_{i}\right)
\end{aligned}
$$


The generators $a_{i}, b_{i}$ are some elements of $\mathfrak{s u}(2,2 \mid 4)$. The operator $d$ is defined by

$$
d \equiv P_{1}+2 P_{2}-P_{3},
$$

with projectors $P_{k}(k=0,1,2,3)$ from $\mathfrak{s u}(2,2 \mid 4)$ to its $\mathbb{Z}_{4}$-graded components $\mathfrak{s u}(2,2 \mid 4)^{(k)}$. In particular, $\mathfrak{s u}(2,2 \mid 4)^{(0)}$ is a gauge symmetry, $\mathfrak{s o}(1,4) \oplus \mathfrak{s o}(5)$.

\subsection{A two-parameter deformation of $\mathrm{AdS}_{5}$}

We consider here a deformation of the $\mathrm{AdS}_{5}$ bosonic part of (2.1) based on the following classical $r$-matrix of Jordanian type,

$$
r_{\text {Jor }}=E_{24} \wedge\left(c_{1} E_{22}-c_{2} E_{44}\right),
$$

where $E_{i j}(i, j=1,2,3,4)$ are the fundamental representation of the generators of $\mathfrak{s u}(2,2)$ defined by $\left(E_{i j}\right)_{k l}=\delta_{i k} \delta_{j l}$. Here $c_{1}$ and $c_{2}$ are constant complex numbers.

To evaluate the action, it is convenient to rewrite the metric part and NS-NS two-form coupled part of the Lagrangian (2.1) into the following form,

$$
\begin{aligned}
L_{G} & =\frac{1}{2} \mathrm{~S} \operatorname{Tr}\left[A_{\tau} P_{2}\left(J_{\tau}\right)-A_{\sigma} P_{2}\left(J_{\sigma}\right)\right], \\
L_{B} & =\frac{1}{2} \mathrm{~S} \operatorname{Tr}\left[A_{\tau} P_{2}\left(J_{\sigma}\right)-A_{\sigma} P_{2}\left(J_{\tau}\right)\right],
\end{aligned}
$$

where $J_{\alpha}$ is a projected current defined as

$$
J_{\alpha} \equiv \frac{1}{1-2\left[R_{\mathrm{Jor}}\right]_{g} \circ P_{2}} A_{\alpha}
$$

Here the parameter $\eta$ in (2.1) is set as $\eta=1$. The linear R-operator $R_{\text {Jor }}$ associated with (2.6) is represented by the identification (2.4).

To find the $\mathrm{AdS}_{5}$ part of (2.7), we use the following parameterization,

$$
g=\exp \left(p_{0} x^{0}+p_{1} x^{1}+p_{2} x^{2}+p_{3} x^{3}\right) \exp \left(\frac{\gamma_{5}}{2} \log z\right) \quad \in \mathrm{SU}(2,2) .
$$

For the $\mathfrak{s u}(2,2)$ generators $p_{\mu}(\mu=0,1,2,3)$ and $\gamma_{5}$, see appendix A. The projected deformed current $P_{2}\left(J_{\alpha}\right)$ is obtained by solving the equation,

$$
\left(1-2 P_{2} \circ R_{g}\right) P_{2}\left(J_{\alpha}\right)=P_{2}\left(A_{\alpha}\right) .
$$

By plugging the projected current $^{1}$

$$
P_{2}\left(A_{\alpha}\right)=\frac{\partial_{\alpha} x^{0} \gamma_{0}+\partial_{\alpha} x^{1} \gamma_{1}+\partial_{\alpha} x^{2} \gamma_{2}+\partial_{\alpha} x^{3} \gamma_{3}+\partial_{\alpha} z \gamma_{5}}{2 z}
$$

with (2.10), the deformed current is evaluated as

$$
P_{2}\left(J_{\alpha}\right)=j_{\alpha}^{0} \gamma_{0}+j_{\alpha}^{1} \gamma_{1}+j_{\alpha}^{2} \gamma_{2}+j_{\alpha}^{3} \gamma_{3}+j_{\alpha}^{5} \gamma_{5},
$$

\footnotetext{
${ }^{1}$ For the convention of $\gamma$-matrices, see appendix A.
} 
with the coefficients

$$
\begin{aligned}
j_{\alpha}^{1}= & \frac{1}{2 z} \partial_{\alpha} x^{1}-\frac{\left(c_{1}+c_{2}\right) x^{1}+i\left(c_{1}-c_{2}\right) x^{2}}{2 \sqrt{2} z^{3}} \partial_{\alpha} x^{+}, \\
j_{\alpha}^{2}= & \frac{1}{2 z} \partial_{\alpha} x^{2}-\frac{\left(c_{1}+c_{2}\right) x^{2}-i\left(c_{1}-c_{2}\right) x^{1}}{2 \sqrt{2} z^{3}} \partial_{\alpha} x^{+}, \\
j_{\alpha}^{0}+j_{\alpha}^{3}= & \frac{1}{\sqrt{2} z} \partial_{\alpha} x^{+}, \\
j_{\alpha}^{0}-j_{\alpha}^{3}= & -\frac{\left(c_{1}+c_{2}\right) x^{1}+i\left(c_{1}-c_{2}\right) x^{2}}{2 z^{3}} \partial_{\alpha} x^{1}-\frac{\left(c_{1}+c_{2}\right) x^{2}-i\left(c_{1}-c_{2}\right) x^{1}}{2 z^{3}} \partial_{\alpha} x^{2} \\
& +\frac{4 c_{1} c_{2}\left(\left(x^{1}\right)^{2}+\left(x^{2}\right)^{2}\right)+\left(c_{1}+c_{2}\right)^{2} z^{2}}{2 \sqrt{2} z^{5}} \partial_{\alpha} x^{+}+\frac{1}{\sqrt{2} z} \partial_{\alpha} x^{-}-\frac{c_{1}+c_{2}}{2 z^{2}} \partial_{\alpha} z, \\
j_{\alpha}^{5}= & \frac{1}{2 z} \partial_{\alpha} z-\frac{c_{1}+c_{2}}{2 \sqrt{2} z^{2}} \partial_{\alpha} x^{+} .
\end{aligned}
$$

Here we have introduced the light-cone coordinates

$$
x^{ \pm} \equiv \frac{x^{0} \pm x^{3}}{\sqrt{2}} .
$$

Finally, the metric part and NS-NS two-form part of the Lagrangian (2.7) are given by

$$
\begin{gathered}
L_{G}=-\gamma^{\alpha \beta}\left[\frac{-2 \partial_{\alpha} x^{+} \partial_{\beta} x^{-}+\partial_{\alpha} x^{1} \partial_{\beta} x^{1}+\partial_{\alpha} x^{2} \partial_{\beta} x^{2}+\partial_{\alpha} z \partial_{\beta} z}{2 z^{2}}\right. \\
\left.-\frac{4 c_{1} c_{2}\left(\left(x^{1}\right)^{2}+\left(x^{2}\right)^{2}\right)+\left(c_{1}+c_{2}\right)^{2} z^{2}}{8 z^{6}} \partial_{\alpha} x^{+} \partial_{\beta} x^{+}\right], \\
L_{B}=-\epsilon^{\alpha \beta}\left[\left(c_{1}+c_{2}\right) \frac{\left(x^{1} \partial_{\alpha} x^{1}+x^{2} \partial_{\alpha} x^{2}+z \partial_{\alpha} z\right) \partial_{\beta} x^{+}}{2 z^{4}}\right. \\
\left.+i\left(c_{1}-c_{2}\right) \frac{\left(x^{2} \partial_{\alpha} x^{1}-x^{1} \partial_{\alpha} x^{2}\right) \partial_{\beta} x^{+}}{2 z^{4}}\right] .
\end{gathered}
$$

Note that the resulting Lagrangian becomes complex in general. Therefore it is necessary to argue the reality condition.

Reality condition. Interestingly, both $L_{G}$ and $L_{B}$ become real if and only if $c_{1}$ and $c_{2}$ are related by the complex conjugation,

$$
c_{1}=c_{2}^{*} \quad \Longleftrightarrow \quad c_{1}=\alpha, \quad c_{2}=\alpha^{*} \quad(\alpha \in \mathbb{C}) .
$$

In particular, the result given in subsection 2.2 of [62] can be reproduced by imposing that

$$
c_{1}=c_{2}=\frac{1}{\sqrt{2}} .
$$

\subsection{The metric and NS-NS two-form}

In the previous subsection, we have seen that a Jordanian $r$-matrix (2.6) yields the metric in the string frame and NS-NS two form, as presented in (2.15). Taking into account the 
reality condition (2.16), the associated metric and NS-NS two form are derived as

$$
\begin{aligned}
d s^{2} & =\frac{-2 d x^{+} d x^{-}+\left(d x^{1}\right)^{2}+\left(d x^{2}\right)^{2}+d z^{2}}{z^{2}}-\frac{|\alpha|^{2}\left(\left(x^{1}\right)^{2}+\left(x^{2}\right)^{2}\right)+(\operatorname{Re}(\alpha))^{2} z^{2}}{z^{6}}\left(d x^{+}\right)^{2}, \\
B_{2} & =-\operatorname{Re}(\alpha) \frac{\left(x^{1} d x^{1}+x^{2} d x^{2}+z d z\right) \wedge d x^{+}}{z^{4}}+\operatorname{Im}(\alpha) \frac{\left(x^{2} d x^{1}-x^{1} d x^{2}\right) \wedge d x^{+}}{z^{4}}
\end{aligned}
$$

It is worth seeing two special cases of $\alpha$ as listed below.

(i) Pure imaginary $\alpha$-deformation. When $\alpha$ is pure imaginary,

$$
\alpha \equiv i \alpha_{\mathrm{I}} \quad \text { with } \quad \alpha_{\mathrm{I}} \in \mathbb{R},
$$

the metric and NS-NS two-form in (2.18) are given by

$$
\begin{aligned}
d s^{2} & =\frac{-2 d x^{+} d x^{-}+\left(d x^{1}\right)^{2}+\left(d x^{2}\right)^{2}+d z^{2}}{z^{2}}-\alpha_{\mathrm{I}}^{2} \frac{\left(x^{1}\right)^{2}+\left(x^{2}\right)^{2}}{z^{6}}\left(d x^{+}\right)^{2}, \\
B_{2} & =\alpha_{\mathrm{I}} \frac{\left(x^{2} d x^{1}-x^{1} d x^{2}\right) \wedge d x^{+}}{z^{4}} .
\end{aligned}
$$

These agree with the ones of the solution found in [61].

(ii) Real $\alpha$-deformation. When $\alpha$ is a real number,

$$
\alpha=\alpha_{\mathrm{R}} \quad \text { with } \quad \alpha_{\mathrm{R}} \in \mathbb{R},
$$

the metric and NS-NS two-form in (2.18) is rewritten as

$$
\begin{aligned}
d s^{2} & =\frac{-2 d x^{+} d x^{-}+\left(d x^{1}\right)^{2}+\left(d x^{2}\right)^{2}+d z^{2}}{z^{2}}-\alpha_{\mathrm{R}}^{2} \frac{\left(x^{1}\right)^{2}+\left(x^{2}\right)^{2}+z^{2}}{z^{6}}\left(d x^{+}\right)^{2}, \\
B_{2} & =-\alpha_{\mathrm{R}} \frac{\left(x^{1} d x^{1}+x^{2} d x^{2}+z d z\right) \wedge d x^{+}}{z^{4}} .
\end{aligned}
$$

The above metric and NS-NS two-form are nothing but the ones obtained in [62].

\section{A chain of dualities for $\operatorname{AdS}_{5} \times \mathrm{S}^{5}$}

In this section, we show that the metric and NS-NS two-form in (2.18) are reproduced by performing TsT-transformations and an S-duality for the $\mathrm{AdS}_{5} \times \mathrm{S}^{5}$ background.

In order to perform T-dualities, let us introduce the polar coordinates on the $x^{1}-x^{2}$ plane,

$$
x^{1}=\rho \cos \varphi, \quad x^{2}=\rho \sin \varphi \quad(0 \leq \rho<\infty, \quad 0 \leq \varphi<2 \pi) .
$$

With these coordinates, the original $\mathrm{AdS}_{5} \times \mathrm{S}^{5}$ background is given by

$$
\begin{aligned}
& d s^{2}=\frac{-2 d x^{+} d x^{-}+d \rho^{2}+\rho^{2} d \varphi^{2}+d z^{2}}{z^{2}}+(d \chi+\omega)^{2}+d s_{\mathbb{C P}^{2}}^{2} \\
& F_{5}=d C_{4}=4\left[-\frac{1}{z^{5}} d x^{+} \wedge d x^{-} \wedge d \rho \wedge \rho d \varphi \wedge d z\right. \\
& \left.\quad+(d \chi+\omega) \wedge d \mu \wedge \sin \mu \Sigma_{1} \wedge \sin \mu \Sigma_{2} \wedge \cos \mu \sin \mu \Sigma_{3}\right], \\
& B_{2}=C_{2}=C=0, \quad \Phi=\Phi_{0} \quad \text { (const.). }
\end{aligned}
$$


Note that the metric of $\mathrm{S}^{5}$ is expressed as a $\mathrm{U}(1)$-fibration over $\mathbb{C P}^{2}$. Here $\chi$ is a local coordinate on the fiber and $\omega$ is a one-form potential for the Kähler form on $\mathbb{C P}^{2}$. In the usual way, the metric of $\mathbb{C P}^{2}$ and $\omega$ are given by

$$
d s_{\mathbb{C P}^{2}}^{2}=d \mu^{2}+\sin ^{2} \mu\left(\Sigma_{1}^{2}+\Sigma_{2}^{2}+\cos ^{2} \mu \Sigma_{3}^{2}\right), \quad \omega \equiv \sin ^{2} \mu \Sigma_{3},
$$

where $\Sigma_{a}(a=1,2,3)$ are defined as

$$
\begin{aligned}
\Sigma_{1} & \equiv \frac{1}{2}(\cos \psi d \theta+\sin \psi \sin \theta d \phi), \quad \Sigma_{2} \equiv \frac{1}{2}(\sin \psi d \theta-\cos \psi \sin \theta d \phi), \\
\Sigma_{3} & \equiv \frac{1}{2}(d \psi+\cos \theta d \phi) .
\end{aligned}
$$

The geometry of $S^{5}$ is described with the five coordinates: $(\chi, \mu, \psi, \theta, \phi)$.

For later computations, it is nice to write down explicitly the R-R four-form $C_{4}$,

$$
C_{4}=\frac{1}{z^{4}} d x^{+} \wedge d x^{-} \wedge d \rho \wedge \rho d \varphi-\sin ^{4} \mu d \chi \wedge \Sigma_{1} \wedge \Sigma_{2} \wedge \Sigma_{3}
$$

Note that the following relations are satisfied,

$$
\Sigma_{1} \wedge \Sigma_{2} \wedge \Sigma_{3}=-\frac{1}{8} \sin \theta d \theta \wedge d \phi \wedge d \psi, \quad d\left(\Sigma_{1} \wedge \Sigma_{2} \wedge \Sigma_{3}\right)=0
$$

It is also helpful to use the relations,

$$
d x^{1} \wedge d x^{2}=d \rho \wedge \rho d \varphi, \quad x^{1} d x^{1}+x^{2} d x^{2}=\rho d \rho, \quad x^{2} d x^{1}-x^{1} d x^{2}=-\rho^{2} d \varphi .
$$

In the following, we will apply TsT transformations and an S-duality for the $\mathrm{AdS}_{5} \times \mathrm{S}^{5}$ background in (3.2).

\subsection{The first TsT transformation}

The first step is to perform a TsT transformation to the $\mathrm{AdS}_{5} \times \mathrm{S}^{5}$ background in (3.2).

Let us first take a T-duality along the $\varphi$-direction in (3.2). According to the rules of T-duality (listed in appendix B), the background is rewritten as

$$
\begin{aligned}
& d \tilde{s}^{2}=\frac{-2 d x^{+} d x^{-}+d \rho^{2}+d z^{2}}{z^{2}}+\frac{z^{2}}{\rho^{2}} d \tilde{\varphi}^{2}+d s_{\mathrm{S}^{5}}^{2} \\
& \tilde{B}_{2}=0, \quad \Phi_{0}-\frac{1}{2} \ln \left(\frac{\rho^{2}}{z^{2}}\right) \\
& \tilde{C}_{3}=-\frac{\rho}{z^{4}} d x^{+} \wedge d x^{-} \wedge d \rho, \quad \tilde{C}_{5}=-\sin ^{4} \mu d \chi \wedge \Sigma_{1} \wedge \Sigma_{2} \wedge \Sigma_{3} \wedge d \tilde{\varphi} .
\end{aligned}
$$

Then the $x^{-}$-coordinate is shifted as

$$
x^{-} \rightarrow x^{-}+a_{1} \tilde{\varphi}
$$


with a real parameter $a_{1}$. The metric and $\tilde{C}_{3}$ are deformed as follows:

$$
\begin{aligned}
d \tilde{s}^{2} & =\frac{-2 d x^{+} d x^{-}+d \rho^{2}+d z^{2}}{z^{2}}-2 \frac{a_{1}}{z^{2}} d x^{+} d \tilde{\varphi}+\frac{z^{2}}{\rho^{2}} d \tilde{\varphi}^{2}+d s_{\mathrm{S}^{5}}^{2}, \\
\tilde{B}_{2} & =0, \quad \tilde{\Phi}=\Phi_{0}-\frac{1}{2} \ln \left(\frac{\rho^{2}}{z^{2}}\right) \\
\tilde{C}_{3} & =-\frac{\rho}{z^{4}} d x^{+} \wedge d x^{-} \wedge d \rho-a_{1} \frac{\rho}{z^{4}} d x^{+} \wedge d \tilde{\varphi} \wedge d \rho, \\
\tilde{C}_{5} & =-\sin ^{4} \mu d \chi \wedge \Sigma_{1} \wedge \Sigma_{2} \wedge \Sigma_{3} \wedge d \tilde{\varphi} .
\end{aligned}
$$

Finally, by taking a T-duality along the $\tilde{\varphi}$-direction, the resulting background is given by

$$
\begin{aligned}
d s^{2} & =\frac{-2 d x^{+} d x^{-}+d \rho^{2}+\rho^{2} d \varphi^{2}+d z^{2}}{z^{2}}-a_{1}^{2} \frac{\rho^{2}}{z^{6}}\left(d x^{+}\right)^{2}+d s_{\mathrm{S}^{5}}^{2}, \\
B_{2} & =-a_{1} \frac{\rho^{2}}{z^{4}} d x^{+} \wedge d \varphi, \quad \Phi=\Phi_{0}, \quad C_{2}=a_{1} \frac{\rho}{z^{4}} d x^{+} \wedge d \rho, \\
C_{4} & =\frac{\rho}{z^{4}} d x^{+} \wedge d x^{-} \wedge d \rho \wedge d \varphi-\sin ^{4} \mu d \chi \wedge \Sigma_{1} \wedge \Sigma_{2} \wedge \Sigma_{3} .
\end{aligned}
$$

Note that the dilaton is constant. Through the coordinate transformations (3.1), the above metric and NS-NS two-form agree with the ones in (2.20) when $a_{1}=-\operatorname{Im}(\alpha)=-\alpha_{\mathrm{I}}$. The NS-NS sector of the background (3.8) has already been obtained in [61].

\subsection{The second TsT transformation}

The next step is to perform another TsT transformation for the background (3.8). This process is essentially the same as the one in [63-65].

Let us first take a T-duality along the $\chi$-direction of $\mathrm{S}^{5}$. The resulting metric is given by

$$
\begin{aligned}
d s^{2} & =\frac{-2 d x^{+} d x^{-}+d \rho^{2}+\rho^{2} d \varphi^{2}+d z^{2}}{z^{2}}-a_{1}^{2} \frac{\rho^{2}}{z^{6}}\left(d x^{+}\right)^{2}+d \tilde{\chi}^{2}+d s_{\mathbb{C P}^{2}}^{2}, \\
\tilde{B}_{2} & =-a_{1} \frac{\rho^{2}}{z^{4}} d x^{+} \wedge d \varphi+\frac{1}{2} \sin ^{2} \mu d \psi \wedge d \tilde{\chi}+\frac{1}{2} \sin ^{2} \mu \cos \theta d \phi \wedge d \tilde{\chi}, \quad \Phi=\Phi_{0}, \\
\tilde{C}_{3} & =a_{1} \frac{\rho}{z^{4}} d x^{+} \wedge d \rho \wedge d \tilde{\chi}-\sin ^{4} \mu \Sigma_{1} \wedge \Sigma_{2} \wedge \Sigma_{3}, \\
\tilde{C}_{5} & =\frac{\rho}{z^{4}} d x^{+} \wedge d x^{-} \wedge d \rho \wedge d \varphi \wedge d \tilde{\chi} .
\end{aligned}
$$

Then, by shifting $x^{-}$as

$$
x^{-} \rightarrow x^{-}+a_{2} \tilde{\chi}
$$

only the metric is deformed as

$$
d s^{2}=\frac{-2 d x^{+} d x^{-}+d \rho^{2}+\rho^{2} d \varphi^{2}+d z^{2}}{z^{2}}-a_{1}^{2} \frac{\rho^{2}}{z^{6}}\left(d x^{+}\right)^{2}-2 \frac{a_{2}}{z^{2}} d x^{+} d \tilde{\chi}+d \tilde{\chi}^{2}+d s_{\mathbb{C P} 2}^{2} .
$$

Finally, by taking a T-duality along the $\tilde{\chi}$-direction, the resulting background is given by

$$
\begin{aligned}
& d s^{2}=\frac{-2 d x^{+} d x^{-}+d \rho^{2}+\rho^{2} d \varphi^{2}+d z^{2}}{z^{2}}-\left(a_{1}^{2} \frac{\rho^{2}}{z^{6}}+a_{2}^{2} \frac{1}{z^{4}}\right)\left(d x^{+}\right)^{2}+(d \chi+\omega)^{2}+d s_{\mathbb{C P}}^{2}, \\
& B_{2}=-a_{1} \frac{\rho^{2}}{z^{4}} d x^{+} \wedge d \varphi-\frac{a_{2}}{z^{2}} d x^{+} \wedge(d \chi+\omega), \quad \Phi=\Phi_{0}, \quad C_{2}=a_{1} \frac{\rho}{z^{4}} d x^{+} \wedge d \rho, \\
& C_{4}=\frac{\rho}{z^{4}} d x^{+} \wedge d x^{-} \wedge d \rho \wedge d \varphi-\sin ^{4} \mu d \chi \wedge \Sigma_{1} \wedge \Sigma_{2} \wedge \Sigma_{3} .
\end{aligned}
$$

As a result, a deformation term of the Schrödinger spacetime has been added. 
When $a_{1}=0$, the Schrödinger solution is realized [63-65]. In comparison to the $r$-matrix (2.6) that is composed of $\mathfrak{s u}(2,2)$ only, the classical $r$-matrix associated with this solution itself is composed of both $\mathfrak{s u}(2,2)$ and $\mathfrak{s u}(4)$ generators because the TsT transformation includes a direction of $S^{5}$. We will explain in very detail the $r$-matrix corresponding to the Schrödinger spacetime in another place [66]. Notably, this result indicates that the Schrödinger spacetime is integrable. ${ }^{2}$ A further remarkable point is that brane-wave type deformations [71] also lead to integrable backgrounds.

\section{$3.3 \quad$ S-duality}

Then let us perform an S-duality for the background (3.11).

The transformation rule of S-duality is given by

$$
\Phi^{\prime}=-\Phi, \quad B_{2}^{\prime}=C_{2}, \quad C_{2}^{\prime}=-B_{2} .
$$

Hence, after performing the S-duality, the resulting background is given by

$$
\begin{aligned}
d s^{2} & =\frac{-2 d x^{+} d x^{-}+d \rho^{2}+\rho^{2} d \varphi^{2}+d z^{2}}{z^{2}}-\left(a_{1}^{2} \frac{\rho^{2}}{z^{6}}+a_{2}^{2} \frac{1}{z^{4}}\right)\left(d x^{+}\right)^{2}+(d \chi+\omega)^{2}+d s_{\mathbb{C P}}^{2}, \\
B_{2} & =a_{1} \frac{\rho}{z^{4}} d x^{+} \wedge d \rho, \quad \Phi=-\Phi_{0}, \quad C_{2}=a_{1} \frac{\rho^{2}}{z^{4}} d x^{+} \wedge d \varphi+\frac{a_{2}}{z^{2}} d x^{+} \wedge(d \chi+\omega), \\
C_{4} & =\frac{\rho}{z^{4}} d x^{+} \wedge d x^{-} \wedge d \rho \wedge d \varphi-\sin ^{4} \mu d \chi \wedge \Sigma_{1} \wedge \Sigma_{2} \wedge \Sigma_{3} .
\end{aligned}
$$

When $a_{1}= \pm a_{2}=\operatorname{Re}(\alpha)=\alpha_{\mathrm{R}}$, the above background agrees with the one in (2.22). This solution is nothing but the one obtained in [62], where the deformation parameter was denoted by $\alpha_{\mathrm{R}} \equiv \eta$. Notably, this result ensures that the solution in [62] is a consistent string background. In particular, the world-sheet beta-function vanishes.

\subsection{The third TsT transformation}

Furthermore, let us perform a TsT-transformation, which is the same as in section 3.1, for the background in (3.13).

The background in (3.13) seems complicated, but it has a U(1) symmetry along the $\varphi$-direction. We first take a T-duality along the $\varphi$-direction. As a result, the background is transformed as

$$
\begin{aligned}
d s^{2} & =\frac{-2 d x^{+} d x^{-}+d \rho^{2}+d z^{2}}{z^{2}}+\frac{z^{2}}{\rho^{2}} d \tilde{\varphi}^{2}-\left(a_{1}^{2} \frac{\rho^{2}}{z^{6}}+a_{2}^{2} \frac{1}{z^{4}}\right)\left(d x^{+}\right)^{2}+(d \chi+\omega)^{2}+d s_{\mathbb{C P}^{2}}^{2} \\
B_{2} & =a_{1} \frac{\rho}{z^{4}} d x^{+} \wedge d \rho, \quad \Phi=-\Phi_{0}-\frac{1}{2} \ln \left(\frac{\rho^{2}}{z^{2}}\right), \quad C_{1}=-a_{1} \frac{\rho^{2}}{z^{4}} d x^{+} \\
C_{3} & =\frac{a_{2}}{z^{2}} d x^{+} \wedge(d \chi+\omega) \wedge d \tilde{\varphi}-\frac{\rho}{z^{4}} d x^{+} \wedge d x^{-} \wedge d \rho \\
C_{5} & =-\sin ^{4} \mu d \chi \wedge \Sigma_{1} \wedge \Sigma_{2} \wedge \Sigma_{3} \wedge d \tilde{\varphi}
\end{aligned}
$$

\footnotetext{
${ }^{2}$ The integrability of Schrödinger spacetimes may be related to the coset structure argued in [67]. A classification of super Schrödinger algebras [68-70] would also play an important role in the future.
} 
Then, by shifting $x^{-}$as

$$
x^{-} \rightarrow x^{-}+a_{3} \tilde{\varphi},
$$

it is rewritten into the following form:

$$
\begin{aligned}
d s^{2}= & \frac{-2 d x^{+} d x^{-}+d \rho^{2}+d z^{2}}{z^{2}}-2 \frac{a_{3}}{z^{2}} d x^{+} d \tilde{\varphi}+\frac{z^{2}}{\rho^{2}} d \tilde{\varphi}^{2}-\left(a_{1}^{2} \frac{\rho^{2}}{z^{6}}+a_{2}^{2} \frac{1}{z^{4}}\right)\left(d x^{+}\right)^{2} \\
& +(d \chi+\omega)^{2}+d s_{\mathbb{C P}}^{2} \\
B_{2}= & a_{1} \frac{\rho}{z^{4}} d x^{+} \wedge d \rho, \quad \Phi=-\Phi_{0}-\frac{1}{2} \ln \left(\frac{\rho^{2}}{z^{2}}\right), \quad C_{1}=-a_{1} \frac{\rho^{2}}{z^{4}} d x^{+} \\
C_{3}= & \frac{a_{2}}{z^{2}} d x^{+} \wedge(d \chi+\omega) \wedge d \tilde{\varphi}-\frac{\rho}{z^{4}} d x^{+} \wedge d x^{-} \wedge d \rho+a_{3} \frac{\rho}{z^{4}} d x^{+} \wedge d \rho \wedge d \tilde{\varphi} \\
C_{5}= & -\sin ^{4} \mu d \chi \wedge \Sigma_{1} \wedge \Sigma_{2} \wedge \Sigma_{3} \wedge d \tilde{\varphi} .
\end{aligned}
$$

Finally, by taking a T-duality along the $\tilde{\varphi}$-direction, the resulting background is given by

$$
\begin{aligned}
d s^{2}= & \frac{-2 d x^{+} d x^{-}+d \rho^{2}+\rho^{2} d \varphi^{2}+d z^{2}}{z^{2}}-\left(\left(a_{1}^{2}+a_{3}^{2}\right) \frac{\rho^{2}}{z^{6}}+a_{2}^{2} \frac{1}{z^{4}}\right)\left(d x^{+}\right)^{2} \\
& +(d \chi+\omega)^{2}+d s_{\mathbb{C P} 2}^{2}, \\
B_{2}= & a_{1} \frac{\rho}{z^{4}} d x^{+} \wedge d \rho-a_{3} \frac{\rho^{2}}{z^{4}} d x^{+} \wedge d \varphi, \quad \Phi=-\Phi_{0}, \\
C_{2}= & a_{1} \frac{\rho^{2}}{z^{4}} d x^{+} \wedge d \varphi+\frac{a_{2}}{z^{2}} d x^{+} \wedge(d \chi+\omega)+a_{3} \frac{\rho}{z^{4}} d x^{+} \wedge d \rho, \\
C_{4}= & \frac{\rho}{z^{4}} d x^{+} \wedge d x^{-} \wedge d \rho \wedge d \varphi-\sin ^{4} \mu d \chi \wedge \Sigma_{1} \wedge \Sigma_{2} \wedge \Sigma_{3} .
\end{aligned}
$$

When $a_{1}= \pm a_{2}=\operatorname{Re}(\alpha)$ and $a_{3}=-\operatorname{Im}(\alpha)$, the solution (3.17) reproduces the metric and NS-NS two-form in (2.18). Note that in this identification the sign concerning $a_{2}$ has not been determined here. In order to fix this ambiguity, we have to perform a supercoset construction.

So far, the R-R sector has not been determined yet from the Yang-Baxter sigma model approach. But the solution (3.17) gives a prediction for the R-R sector. If the gravity/CYBE correspondence is true, then the R-R sector of (3.17) should be reproduced by performing a supercoset construction. We will not try to do that here and leave it as a future problem. However, it should be derived as expected. The kappa-invariance of the deformed string action would also imply the agreement.

\section{Duality-chains and classical $r$-matrices}

It is worth summarizing the relation between duality-chains and classical $r$-matrices. This is nothing but the gravity/CYBE correspondence concerned with the present scope.

For the completeness, we first consider the fourth TsT-transformation in subsection 4.1 and the second S-duality in subsection 4.2. In subsection 4.3, we propose a classical $r$-matrix including three parameters and summarize a concrete realization of the gravity/CYBE correspondence. 


\subsection{The fourth TsT transformation}

Let us consider here a TsT-transformation for the background in (3.17).

We first perform a T-duality for the background in (3.17) along the $\chi$-direction. The resulting background is

$$
\begin{aligned}
& d s^{2}=d s_{\mathrm{AdS}_{5}}^{2}-\left(\left(a_{1}^{2}+a_{3}^{2}\right) \frac{\rho^{2}}{z^{6}}+a_{2}^{2} \frac{1}{z^{4}}\right)\left(d x^{+}\right)^{2}+d \tilde{\chi}^{2}+d s_{\mathbb{C P}^{2}}^{2}, \\
& B_{2}=d x^{+} \wedge\left(a_{1} \frac{\rho d \rho}{z^{4}}-a_{3} \frac{\rho^{2} d \varphi}{z^{4}}\right)+\omega \wedge d \tilde{\chi}, \quad \tilde{\Phi}=-\Phi_{0}, \quad C_{1}=-\frac{a_{2}}{z^{2}} d x^{+}, \\
& C_{3}=d x^{+} \wedge\left(a_{1} \frac{\rho^{2} d \varphi}{z^{4}}+a_{3} \frac{\rho d \rho}{z^{4}}\right) \wedge \tilde{d} \chi-\sin ^{4} \mu \Sigma_{1} \wedge \Sigma_{2} \wedge \Sigma_{3}, \\
& C_{5}=\frac{\rho}{z^{4}} d x^{+} \wedge d x^{-} \wedge d \rho \wedge d \phi \wedge d \tilde{\chi},
\end{aligned}
$$

where the undeformed metric of $\mathrm{AdS}_{5}$ is denoted as

$$
d s_{\mathrm{AdS}_{5}}^{2}=\frac{-2 d x^{+} d x^{-}+d \rho^{2}+\rho^{2} d \varphi^{2}+d z^{2}}{z^{2}} .
$$

Then, by shifting the $x^{-}$-coordinate as

$$
x^{-} \rightarrow x^{-}+a_{4} \tilde{\chi},
$$

only the metric in (4.1) is modified as

$$
d s^{2}=d s_{\mathrm{AdS}_{5}}^{2}-\frac{2 a_{4}}{z^{2}} d x^{+} d \tilde{\chi}-\left(\left(a_{1}^{2}+a_{3}^{2}\right) \frac{\rho^{2}}{z^{6}}+a_{2}^{2} \frac{1}{z^{4}}\right)\left(d x^{+}\right)^{2}+d \tilde{\chi}^{2}+d s_{\mathbb{C P}^{2}}^{2}
$$

Finally, by T-dualizing back along the $\tilde{\chi}$-direction, we arrive at the following background:

$$
\begin{aligned}
& d s^{2}=d s_{\mathrm{AdS}_{5}}^{2}-\left(\left(a_{1}^{2}+a_{3}^{2}\right) \frac{\rho^{2}}{z^{6}}+\left(a_{2}^{2}+a_{4}^{2}\right) \frac{1}{z^{4}}\right)\left(d x^{+}\right)^{2}+(d \chi+\omega)^{2}+d s_{\mathbb{C P}^{2}}^{2}, \\
& B_{2}=d x^{+} \wedge\left(-a_{3} \frac{\rho^{2} d \varphi}{z^{4}}-a_{4} \frac{d \chi+\omega}{z^{2}}+a_{1} \frac{\rho d \rho}{z^{4}}\right), \quad \Phi=-\Phi_{0}, \\
& C_{2}=d x^{+} \wedge\left(a_{1} \frac{\rho^{2} d \varphi}{z^{4}}+a_{2} \frac{d \chi+\omega}{z^{2}}+a_{3} \frac{\rho d \rho}{z^{4}}\right), \\
& C_{4}=\frac{\rho}{z^{4}} d x^{+} \wedge d x^{-} \wedge d \rho \wedge d \varphi-\sin ^{4} \mu d \chi \wedge \Sigma_{1} \wedge \Sigma_{2} \wedge \Sigma_{3} .
\end{aligned}
$$

\subsection{The second S-duality}

One may notice that, in the background (4.5), the NS-NS two-form $B_{2}$ and the RamondRamond two-form $C_{2}$ are turned on in a symmetric way. This is because the background (4.5) is obtained by two same TsT-transformations before and after the Sduality (3.12). Then, let us consider the second S-duality of (4.5). The resulting back- 
ground is given by

$$
\begin{aligned}
d s^{2} & =d s_{\mathrm{AdS}_{5}}^{2}-\left(\left(a_{1}^{2}+a_{3}^{2}\right) \frac{\rho^{2}}{z^{6}}+\left(a_{2}^{2}+a_{4}^{2}\right) \frac{1}{z^{4}}\right)\left(d x^{+}\right)^{2}+(d \chi+\omega)^{2}+d s_{\mathbb{C P}^{2}}^{2}, \\
B_{2} & =d x^{+} \wedge\left(a_{1} \frac{\rho^{2} d \varphi}{z^{4}}+a_{2} \frac{d \chi+\omega}{z^{2}}+a_{3} \frac{\rho d \rho}{z^{4}}\right), \quad \Phi=\Phi_{0}, \\
C_{2} & =d x^{+} \wedge\left(a_{3} \frac{\rho^{2} d \varphi}{z^{4}}+a_{4} \frac{d \chi+\omega}{z^{2}}-a_{1} \frac{\rho d \rho}{z^{4}}\right), \\
C_{4} & =\frac{\rho}{z^{4}} d x^{+} \wedge d x^{-} \wedge d \rho \wedge d \varphi-\sin ^{4} \mu d \chi \wedge \Sigma_{1} \wedge \Sigma_{2} \wedge \Sigma_{3} .
\end{aligned}
$$

\subsection{A three-parameter deformation of $\mathrm{AdS}_{5} \times \mathrm{S}^{5}$}

Now it is turn to consider a classical $r$-matrix corresponding to the background (4.6). It is argued to be the following form,

$$
r_{12}\left(b_{1}, b_{2}, b_{3}\right)=\frac{1}{\sqrt{2} i} E_{24} \wedge\left(b_{1}\left(E_{22}+E_{44}\right)+\frac{b_{2}}{2}\left(h_{4}+h_{5}+h_{6}\right)+i b_{3}\left(E_{22}-E_{44}\right)\right) .
$$

Here $h_{4}, h_{5}$ and $h_{6}$ are the three Cartan generators ${ }^{3}$ of $\mathfrak{s u}(4)$ rather than $\mathfrak{s u}(2,2)$, and $b_{1}, b_{2}, b_{3}$ are real deformation parameters. A direct computation shows that this is a solution of the CYBE.

Plugging the classical $r$-matrix (4.7) with the classical action (2.1), the resulting metric and NS-NS two-form turn out to be

$$
\begin{aligned}
d s^{2} & =d s_{\mathrm{AdS}_{5}}^{2}+d s_{\mathrm{S}^{5}}^{2}-\frac{\left(b_{1}^{2}+b_{3}^{2}\right) \rho^{2}+\left(b_{2}^{2}+b_{3}^{2}\right) z^{2}}{z^{6}}\left(d x^{+}\right)^{2}, \\
B_{2} & =d x^{+} \wedge\left(-b_{1} \frac{\rho^{2} d \varphi}{z^{4}}-b_{2} \frac{d \chi+\omega}{z^{2}}+b_{3} \frac{\rho d \rho}{z^{4}}\right),
\end{aligned}
$$

where $d s_{\mathrm{AdS}_{5}}^{2}$ and $d s_{\mathrm{S}^{5}}^{2}$ are the undeformed metrics of $\mathrm{AdS}_{5}$ and $\mathrm{S}^{5}$, respectively.

Indeed, the above metric and NS-NS two-form agree with the ones obtained by the fourth TsT-transformation in (4.5) and the S-duality in (4.6) with the following parameter identifications, respectively,

$$
\begin{array}{lll}
b_{1}=a_{3}, & b_{2}=a_{4}, & b_{3}=a_{1}= \pm a_{2} \\
b_{1}=-a_{1}, & b_{2}=-a_{2}, & b_{3}=a_{3}= \pm a_{4}
\end{array} \quad \text { for } \quad \text { for } \quad \text { (4.6). }
$$

It is worth comparing the above results with the deformed backgrounds from TsTtransformations and an S-duality in section 3. The comprehensive relations are summarized in table 1. Here the symbol (TsT) $)_{\varphi}^{a_{1}}$, for instance, stands for a TsT-transformation consisting of a T-duality for the $\varphi$-direction and the shift $x^{-} \rightarrow x^{-}+a_{1} \tilde{\varphi}$. The capital S denotes an S-duality. For the details, see section 3.

\footnotetext{
${ }^{3}$ The convention of the $\mathfrak{s u}(4)$ algebra would be presented in [66]. The case with $b_{1}=b_{3}=0$ will be elaborated in [66].
} 


\begin{tabular}{|c|c|c|}
\hline Classical $r$-matrices & Duality chains & Backgrounds \\
\hline$r_{12}\left(a_{1}, 0,0\right)$ & $(\mathrm{TsT})_{\varphi}^{a_{1}}$ & $(3.8)$, see also $[61]$ \\
\hline$r_{12}\left(a_{1}, a_{2}, 0\right)$ & $(\mathrm{TsT})_{\chi}^{a_{2}} \circ(\mathrm{TsT})_{\varphi}^{a_{1}}$ & $(3.11)$ \\
\hline$r_{12}\left(0,0, a_{1}\right)$ & $\mathrm{So}(\mathrm{TsT})_{\chi}^{ \pm a_{1}} \circ(\mathrm{TsT})_{\varphi}^{a_{1}}$ & $(3.13)$, see also $[62]$ \\
\hline$r_{12}\left(a_{3}, 0, a_{1}\right)$ & 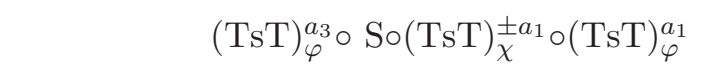 & $(3.17)$ \\
\hline$r_{12}\left(a_{3}, a_{4}, a_{1}\right)$ & 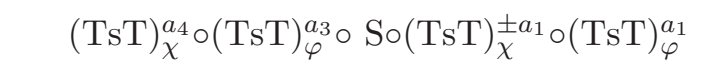 & $(4.5)$ \\
\hline$r_{12}\left(-a_{1},-a_{2}, a_{3}\right)$ & $\mathrm{So}(\mathrm{TsT})_{\chi}^{ \pm a_{3}} \circ(\mathrm{TsT})_{\varphi}^{a_{3}} \circ \mathrm{S} \circ(\mathrm{TsT})_{\chi}^{a_{2}} \circ(\mathrm{TsT})_{\varphi}^{a_{1}}$ & $(4.6)$ \\
\hline
\end{tabular}

Table 1. The classical $r$-matrix in (4.7) and the associated duality chains.

As for table 1, note that the following duality chains including S-dualities are realized only if some parameter constraints are satisfied;

$$
\begin{aligned}
\mathrm{S} \circ(\mathrm{TsT})_{\chi}^{a_{2}} \circ(\mathrm{TsT})_{\varphi}^{a_{1}} & \text { if } & a_{2}= \pm a_{1}, \\
(\mathrm{TsT})_{\varphi}^{a_{3}} \circ \mathrm{S} \circ(\mathrm{TsT})_{\chi}^{a_{2}} \circ(\mathrm{TsT})_{\varphi}^{a_{1}} & \text { if } & a_{2}= \pm a_{1}, \\
(\mathrm{TsT})_{\chi}^{a_{4}} \circ(\mathrm{TsT})_{\varphi}^{a_{3}} \circ \mathrm{S} \circ(\mathrm{TsT})_{\chi}^{a_{2}} \circ(\mathrm{TsT})_{\varphi}^{a_{1}} & \text { if } & a_{2}= \pm a_{1}, \\
\mathrm{~S} \circ(\mathrm{TsT})_{\chi}^{a_{4}} \circ(\mathrm{TsT})_{\varphi}^{a_{3}} \circ \mathrm{S} \circ(\mathrm{TsT})_{\chi}^{a_{2}} \circ(\mathrm{TsT})_{\varphi}^{a_{1}} & \text { if } & a_{4}= \pm a_{3} .
\end{aligned}
$$

In order to see the correspondence between the $r$-matrix in (4.7) and deformed backgrounds, we need to impose constraints for the values of $a_{1}, a_{2}, a_{3}, a_{4}$. It seems likely that the constraints would come from the S-duality, but at the present moment we have no idea for the origin. To reveal it, it would be important to develop Yang-Baxter deformations of the D-string action. Then the origin may be understood as a consistency condition of the S-duality transformation.

\section{Conclusion and discussion}

We have further studied integrable deformations of the $\mathrm{AdS}_{5} \times \mathrm{S}^{5}$ superstring based on the Yang-Baxter sigma model approach with classical $r$-matrices satisfying the CYBE. By focusing upon a classical $r$-matrix with two parameters and its three-parameter generalization, we have presented a family of the deformed metric and NS-NS two-form. The corresponding solutions of type IIB supergravity have been successfully obtained by performing TsT-transformations and S-dualities. In particular, it includes the solution found by Hubeny-Rangamani-Ross [61] and the one found by us [62] as special cases.

So far, we have seen that a certain multi-parameter family of classical $r$-matrices correspond to a chain of TsT-transformations and S-dualities for the undeformed $\mathrm{AdS}_{5} \times \mathrm{S}^{5}$ background. It seems likely that Yang-Baxter sigma models based on CYBE could reproduce duality chains of $\mathrm{AdS}_{5} \times \mathrm{S}^{5}$. In fact, three-parameter $\gamma$-deformations of $\mathrm{S}^{5}[49,50]$ and gravity duals for noncommutative gauge theories [52-55] have been reproduced from the associated classical $r$-matrices [51, 56]. Integrable deformations based on (at least) a certain class of $r$-matrices can be undone with non-local gauge transformations and twisted boundary condition, by following the philosophy of [72]. 
So far, we have discussed duality chains of $\mathrm{AdS}_{5} \times \mathrm{S}^{5}$. This is a simple story and one may think of that Yang-Baxter sigma models would work only for duality chains. However, this is not the case. For example, more complicated backgrounds have been presented in [62] from the Yang-Baxter sigma model approach and those do not seem to be realized as TsT transformations of $\mathrm{AdS}_{5} \times \mathrm{S}^{5}$ because of the singular structure. Therefore, it seems likely that the Yang-Baxter sigma model approach would include a wider class of gravity solutions which cannot be realized by performing string dualities for the undeformed $\operatorname{AdS}_{5} \times \mathrm{S}^{5}$.

It would be of great importance to clarify the applicability of Yang-Baxter sigma models.

\section{Acknowledgments}

We are very grateful to Io Kawaguchi for collaboration at the very early stage of this work. We appreciate P. Marcos Crichigno, Minkyo Kim, Marc Magro and Benoit Vicedo for valuable comments and discussions. This work is supported in part by JSPS JapanHungary Research Cooperative Program.

\section{A Notation and convention}

An explicit basis of $\mathfrak{s u}(2,2)$ is represented by the following $\gamma$-matrices,

$$
\begin{aligned}
\gamma_{1} & =\left[\begin{array}{cccc}
0 & 0 & 0 & -1 \\
0 & 0 & 1 & 0 \\
0 & 1 & 0 & 0 \\
-1 & 0 & 0 & 0
\end{array}\right], \quad \gamma_{2}=\left[\begin{array}{cccc}
0 & 0 & 0 & i \\
0 & 0 & i & 0 \\
0 & -i & 0 & 0 \\
-i & 0 & 0 & 0
\end{array}\right], \quad \gamma_{3}=\left[\begin{array}{cccc}
0 & 0 & 1 & 0 \\
0 & 0 & 0 & 1 \\
1 & 0 & 0 & 0 \\
0 & 1 & 0 & 0
\end{array}\right], \\
\gamma_{0} & =\left[\begin{array}{cccc}
0 & 0 & 1 & 0 \\
0 & 0 & 0 & -1 \\
-1 & 0 & 0 & 0 \\
0 & 1 & 0 & 0
\end{array}\right], \quad \gamma_{5}=i \gamma_{1} \gamma_{2} \gamma_{3} \gamma_{0}=\left[\begin{array}{cccc}
1 & 0 & 0 & 0 \\
0 & 1 & 0 & 0 \\
0 & 0 & -1 & 0 \\
0 & 0 & 0 & -1
\end{array}\right] .
\end{aligned}
$$

With the Lorentzian metric $\eta_{\mu \nu}=\operatorname{diag}(-1,1,1,1)$, the Clifford algebra is satisfied as

$$
\left\{\gamma_{\mu}, \gamma_{\nu}\right\}=2 \eta_{\mu \nu}, \quad\left\{\gamma_{\mu}, \gamma_{5}\right\}=0, \quad\left(\gamma_{5}\right)^{2}=1
$$

The Lie algebra $\mathfrak{s o}(1,4)$ is formed by the generators

$$
m_{\mu \nu}=\frac{1}{4}\left[\gamma_{\mu}, \gamma_{\nu}\right], \quad m_{\mu 5}=\frac{1}{4}\left[\gamma_{\mu}, \gamma_{5}\right] \quad(\mu, \nu=0,1,2,3),
$$

and then enlarged algebra $\mathfrak{s o}(2,4)=\mathfrak{s u}(2,2)$ is spanned by the following set:

$$
m_{\mu \nu}, \quad m_{\mu 5}, \quad \frac{1}{2} \gamma_{\mu}, \quad \frac{1}{2} \gamma_{5} .
$$

The reality condition for these generators are given by

$$
M^{\dagger} \gamma_{0}+\gamma_{0} M=0 \quad \text { for } \quad{ }^{\forall} M \in \mathfrak{s u}(2,2) .
$$


The generators $p_{\mu}$ used to parameterize $g \in \mathrm{SU}(2,2)$ in $(2.9)$ are defined as

$$
p_{\mu} \equiv \frac{1}{2} \gamma_{\mu}-m_{\mu 5}
$$

To see the $\mathfrak{s o}(2,4)$ algebra explicitly, one can introduce the generators as follows:

$$
\begin{aligned}
\tilde{m}_{\mu \nu} & =\frac{1}{4}\left[\gamma_{\mu}, \gamma_{\nu}\right], & \tilde{m}_{\mu 5} & =\frac{1}{4}\left[\gamma_{\mu}, \gamma_{5}\right] \quad(\mu, \nu=0,1,2,3), \\
\tilde{m}_{\mu,-1} & =-\tilde{m}_{-1, \mu}=\frac{1}{2} \gamma_{\mu}, & \tilde{m}_{5,-1} & =-\tilde{m}_{-1,5}=\frac{1}{2} \gamma_{5} .
\end{aligned}
$$

Indeed, these generators satisfy the commutation relations,

$$
\left[\tilde{m}_{\hat{\mu} \hat{\nu}}, \tilde{m}_{\hat{\rho} \hat{\sigma}}\right]=\tilde{\eta}_{\hat{\rho} \hat{\nu}} \tilde{m}_{\hat{\mu} \hat{\sigma}}-\tilde{\eta}_{\hat{\rho} \hat{\mu}} \tilde{m}_{\hat{\nu} \hat{\sigma}}-\tilde{\eta}_{\hat{\sigma} \hat{\nu}} \tilde{m}_{\hat{\mu} \hat{\rho}}+\tilde{\eta}_{\hat{\sigma} \hat{\mu}} \tilde{m}_{\hat{\nu} \hat{\rho}}
$$

Here $\hat{\mu}, \hat{\nu}, \hat{\rho}, \hat{\sigma}=-1,0,1,2,3,5$ and $\tilde{\eta}_{\hat{\mu} \hat{\nu}}=\operatorname{diag}(-1,-1,1,1,1,1)$.

\section{B The T-duality rules}

The rules of T-duality [73-75] are summarized here. We basically follow appendix C of [75].

The transformation rules between type IIB and type IIA supergravities are listed below. Note that the T-duality is performed for the $y$-direction and the other coordinates are denoted by $a, b, a_{i}(i=1, \ldots)$. The fields of type IIB supergravity are the metric $g_{\mu \nu}$, NS-NS two-form $B_{2}$, dilaton $\Phi$, R-R gauge fields $C^{(2 n)}$. The ones of type IIA supergravity are denoted with the tilde, the metric $\tilde{g}_{\mu \nu}$, NS-NS two-form $\tilde{B}_{2}$, dilaton $\tilde{\Phi}$, and R-R gauge fields $\tilde{C}^{(2 n+1)}$.

\section{From type IIB to type IIA:}

$$
\begin{aligned}
\tilde{g}_{y y} & =\frac{1}{g_{y y}}, \quad \tilde{g}_{a y}=\frac{B_{a y}}{g_{y y}}, \quad \tilde{g}_{a b}=g_{a b}-\frac{g_{y a} g_{y b}-B_{y a} B_{y b}}{g_{y y}}, \\
\tilde{B}_{a y} & =\frac{g_{a y}}{g_{y y}}, \quad \tilde{B}_{a b}=B_{a b}-\frac{g_{y a} B_{y b}-B_{y a} g_{y b}}{g_{y y}}, \quad \tilde{\Phi}=\Phi-\frac{1}{2} \ln g_{y y}, \\
\tilde{C}_{a_{1} \cdots a_{2 n+1}}^{(2 n+1)} & =-C_{a_{1} \cdots a_{2 n+1}}^{(2 n+2)}-(2 n+1) B_{y\left[a_{1}\right.} C_{\left.a_{2} \cdots a_{2 n+1}\right]}^{(2 n)}+2 n(2 n+1) \frac{B_{y\left[a_{1}\right.} g_{a_{2}|y|} C_{\left.a_{3} \cdots a_{2 n+1}\right] y}^{(2 n)}}{g_{y y}}, \\
\tilde{C}_{a_{1} \cdots a_{2 n} y}^{(2 n+1)} & =C_{a_{1} \cdots a_{2 n}}^{(2 n)}+2 n \frac{g_{y\left[a_{1}\right.} C_{\left.a_{2} \cdots a_{2 n}\right] y}^{(2 n)} .}{g_{y y}} .
\end{aligned}
$$

where the anti-symmetrization for indices is defined as, for example,

$$
A_{[a} B_{b]} \equiv \frac{1}{2}\left(A_{a} B_{b}-A_{b} B_{a}\right)
$$

The symbol $|y|$ inside the anti-symmetrization means that the indices other than the index $y$ are anti-symmetrized. 


\section{From type IIA to type IIB:}

$$
\begin{aligned}
g_{y y} & =\frac{1}{\tilde{g}_{y y}}, \quad g_{a y}=\frac{\tilde{B}_{a y}}{\tilde{g}_{y y}}, \quad g_{a b}=\tilde{g}_{a b}-\frac{\tilde{g}_{y a} \tilde{g}_{y b}-\tilde{B}_{y a} \tilde{B}_{y b}}{\tilde{g}_{y y}}, \\
B_{a y} & =\frac{\tilde{g}_{a y}}{\tilde{g}_{y y}}, \quad B_{a b}=\tilde{B}_{a b}-\frac{\tilde{g}_{y a} \tilde{B}_{y b}-\tilde{B}_{y a} \tilde{g}_{y b}}{\tilde{g}_{y y}}, \quad \Phi=\tilde{\Phi}-\frac{1}{2} \ln \tilde{g}_{y y}, \\
C_{a_{1} \cdots a_{2 n}}^{(2 n)} & =\tilde{C}_{a_{1} \cdots a_{2 n} y}^{(2 n+1)}-2 n \tilde{B}_{y\left[a_{1}\right.} \tilde{C}_{\left.a_{2} \cdots a_{2 n}\right]}^{(2 n-1)}+2 n(2 n-1) \frac{\tilde{B}_{y\left[a_{1}\right.} \tilde{g}_{a_{2}|y|} \tilde{C}_{\left.a_{3} \cdots a_{2 n}\right] y}^{(2 n-1)}}{\tilde{g}_{y y}} \\
C_{a_{1} \cdots a_{2 n-1} y}^{(2 n)} & =-\tilde{C}_{a_{1} \cdots a_{2 n-1}}^{(2 n-1)}-(2 n-1) \frac{\tilde{g}_{y\left[a_{1}\right.} \tilde{C}_{\left.a_{2} \cdots a_{2 n-1}\right] y}^{(2 n-1)}}{\tilde{g}_{y y}} .
\end{aligned}
$$

Open Access. This article is distributed under the terms of the Creative Commons Attribution License (CC-BY 4.0), which permits any use, distribution and reproduction in any medium, provided the original author(s) and source are credited.

\section{References}

[1] J.M. Maldacena, The large- $N$ limit of superconformal field theories and supergravity, Int. J. Theor. Phys. 38 (1999) 1113 [hep-th/9711200] [INSPIRE].

[2] N. Beisert et al., Review of AdS/CFT integrability: an overview, Lett. Math. Phys. 99 (2012) 3 [arXiv:1012.3982] [INSPIRE].

[3] R.R. Metsaev and A.A. Tseytlin, Type IIB superstring action in $A d S_{5} \times S^{5}$ background, Nucl. Phys. B 533 (1998) 109 [hep-th/9805028] [INSPIRE].

[4] I. Bena, J. Polchinski and R. Roiban, Hidden symmetries of the $A d S_{5} \times S^{5}$ superstring, Phys. Rev. D 69 (2004) 046002 [hep-th/0305116] [INSPIRE].

[5] R. Roiban and W. Siegel, Superstrings on $A d S_{5} \times S^{5}$ supertwistor space, JHEP 11 (2000) 024 [hep-th/0010104] [INSPIRE].

[6] M. Hatsuda and K. Yoshida, Classical integrability and super Yangian of superstring on $A d S_{5} \times S^{5}, A d v$. Theor. Math. Phys. 9 (2005) 703 [hep-th/0407044] [INSPIRE].

[7] M. Hatsuda and K. Yoshida, Super Yangian of superstring on $A d S_{5} \times S^{5}$ revisited, Adv. Theor. Math. Phys. 15 (2011) 1485 [arXiv:1107.4673] [INSPIRE].

[8] K. Zarembo, Strings on semisymmetric superspaces, JHEP 05 (2010) 002 [arXiv: 1003.0465] [INSPIRE].

[9] L. Wulff, Superisometries and integrability of superstrings, JHEP 05 (2014) 115 [arXiv: 1402.3122] [INSPIRE].

[10] I. Kawaguchi and K. Yoshida, Hidden Yangian symmetry in $\sigma$-model on squashed sphere, JHEP 11 (2010) 032 [arXiv: 1008.0776] [INSPIRE].

[11] I. Kawaguchi and K. Yoshida, Hybrid classical integrability in squashed $\sigma$-models, Phys. Lett. B 705 (2011) 251 [arXiv:1107.3662] [INSPIRE].

[12] I. Kawaguchi and K. Yoshida, Hybrid classical integrable structure of squashed $\sigma$-models: a short summary, J. Phys. Conf. Ser. 343 (2012) 012055 [arXiv:1110.6748] [INSPIRE]. 
[13] I. Kawaguchi, T. Matsumoto and K. Yoshida, The classical origin of quantum affine algebra in squashed $\sigma$-models, JHEP 04 (2012) 115 [arXiv:1201.3058] [INSPIRE].

[14] I. Kawaguchi, T. Matsumoto and K. Yoshida, On the classical equivalence of monodromy matrices in squashed $\sigma$-model, JHEP 06 (2012) 082 [arXiv:1203.3400] [INSPIRE].

[15] I. Kawaguchi and K. Yoshida, Classical integrability of Schrödinger $\sigma$-models and q-deformed Poincaré symmetry, JHEP 11 (2011) 094 [arXiv: 1109.0872] [INSPIRE].

[16] I. Kawaguchi and K. Yoshida, Exotic symmetry and monodromy equivalence in Schrödinger $\sigma$-models, JHEP 02 (2013) 024 [arXiv: 1209.4147] [INSPIRE].

[17] I. Kawaguchi, T. Matsumoto and K. Yoshida, Schrödinger $\sigma$-models and Jordanian twists, JHEP 08 (2013) 013 [arXiv: 1305.6556] [INSPIRE].

[18] D. Orlando, S. Reffert and L.I. Uruchurtu, Classical integrability of the squashed three-sphere, warped $A d S_{3}$ and Schrödinger spacetime via T-duality, J. Phys. A 44 (2011) 115401 [arXiv:1011.1771] [INSPIRE].

[19] B. Basso and A. Rej, On the integrability of two-dimensional models with $\mathrm{U}(1) \times \mathrm{SU}(N)$ symmetry, Nucl. Phys. B 866 (2013) 337 [arXiv:1207.0413] [INSPIRE].

[20] I. Kawaguchi, D. Orlando and K. Yoshida, Yangian symmetry in deformed WZNW models on squashed spheres, Phys. Lett. B 701 (2011) 475 [arXiv:1104.0738] [InSPIRE].

[21] I. Kawaguchi and K. Yoshida, A deformation of quantum affine algebra in squashed Wess-Zumino-Novikov-Witten models, J. Math. Phys. 55 (2014) 062302 [arXiv:1311.4696] [INSPIRE].

[22] F. Delduc, M. Magro and B. Vicedo, Integrable double deformation of the principal chiral model, Nucl. Phys. B 891 (2015) 312 [arXiv:1410.8066] [INSPIRE].

[23] T.J. Hollowood, J.L. Miramontes and D.M. Schmidtt, Integrable deformations of strings on symmetric spaces, JHEP 11 (2014) 009 [arXiv:1407.2840] [INSPIRE].

[24] T.J. Hollowood, J.L. Miramontes and D.M. Schmidtt, An integrable deformation of the $A d S_{5} \times S^{5}$ superstring, J. Phys. A 47 (2014) 495402 [arXiv:1409.1538] [InSPIRE].

[25] K. Sfetsos, Integrable interpolations: from exact CFTs to non-abelian T-duals, Nucl. Phys. B 880 (2014) 225 [arXiv:1312.4560] [InSPIRE].

[26] G. Itsios, K. Sfetsos, K. Siampos and A. Torrielli, The classical Yang-Baxter equation and the associated Yangian symmetry of gauged WZW-type theories,

Nucl. Phys. B 889 (2014) 64 [arXiv:1409.0554] [INSPIRE].

[27] C. Klimčík, Yang-Baxter $\sigma$-models and dS/AdS T duality, JHEP 12 (2002) 051 [hep-th/0210095] [INSPIRE].

[28] C. Klimčík, On integrability of the Yang-Baxter $\sigma$-model, J. Math. Phys. 50 (2009) 043508 [arXiv:0802.3518] [INSPIRE].

[29] C. Klimčík, Integrability of the bi-Yang-Baxter $\sigma$-model, Lett. Math. Phys. 104 (2014) 1095 [arXiv: 1402.2105] [INSPIRE].

[30] R. Squellari, Yang-Baxter $\sigma$ model: quantum aspects, Nucl. Phys. B 881 (2014) 502 [arXiv: 1401.3197] [INSPIRE].

[31] F. Delduc, M. Magro and B. Vicedo, On classical q-deformations of integrable $\sigma$-models, JHEP 11 (2013) 192 [arXiv:1308.3581] [INSPIRE]. 
[32] F. Delduc, M. Magro and B. Vicedo, An integrable deformation of the $A d S_{5} \times S^{5}$ superstring action, Phys. Rev. Lett. 112 (2014) 051601 [arXiv: 1309.5850] [INSPIRE].

[33] F. Delduc, M. Magro and B. Vicedo, Derivation of the action and symmetries of the q-deformed $A d S_{5} \times S^{5}$ superstring, JHEP 10 (2014) 132 [arXiv:1406.6286] [INSPIRE].

[34] G. Arutyunov, R. Borsato and S. Frolov, S-matrix for strings on $\eta$-deformed $A d S_{5} \times S^{5}$, JHEP 04 (2014) 002 [arXiv: 1312.3542] [INSPIRE].

[35] B. Hoare, R. Roiban and A.A. Tseytlin, On deformations of $A d S_{n} \times S^{n}$ supercosets, JHEP 06 (2014) 002 [arXiv: 1403.5517] [INSPIRE].

[36] G. Arutyunov, M. de Leeuw and S.J. van Tongeren, The exact spectrum and mirror duality of the $\left(A d S_{5} \times S^{5}\right)_{\eta}$ superstring, Theor. Math. Phys. 182 (2015) 23 [arXiv:1403.6104] [INSPIRE].

[37] G. Arutyunov and S.J. van Tongeren, $A d S_{5} \times S^{5}$ mirror model as a string $\sigma$-model, Phys. Rev. Lett. 113 (2014) 261605 [arXiv:1406.2304] [InSPIRE].

[38] T. Kameyama and K. Yoshida, Anisotropic Landau-Lifshitz $\sigma$-models from q-deformed $A d S_{5} \times S^{5}$ superstrings, JHEP 08 (2014) 110 [arXiv: 1405.4467] [INSPIRE].

[39] T. Kameyama and K. Yoshida, String theories on warped AdS backgrounds and integrable deformations of spin chains, JHEP 05 (2013) 146 [arXiv:1304.1286] [INSPIRE].

[40] M. Khouchen and J. Kluson, Giant magnon on deformed $A d S_{3} \times S^{3}$, Phys. Rev. D 90 (2014) 066001 [arXiv: 1405.5017] [InSPIRE].

[41] C. Ahn and P. Bozhilov, Finite-size giant magnons on $\eta$-deformed $A d S_{5} \times S^{5}$, Phys. Lett. B 737 (2014) 293 [arXiv: 1406.0628] [INSPIRE].

[42] A. Banerjee and K.L. Panigrahi, On the rotating and oscillating strings in $\left(A d S_{3} \times S^{3}\right)_{\kappa}$, JHEP 09 (2014) 048 [arXiv: 1406.3642] [INSPIRE].

[43] G. Arutyunov and D. Medina-Rincon, Deformed Neumann model from spinning strings on $\left(A d S_{5} \times S^{5}\right)_{\eta}, J H E P 1410(2014) 50$ [arXiv:1406.2536] [INSPIRE].

[44] T. Kameyama and K. Yoshida, A new coordinate system for $q$-deformed $A d S_{5} \times S^{5}$ and classical string solutions, J. Phys. A 48 (2015) 075401 [arXiv: 1408.2189] [InSPIRE].

[45] T. Kameyama and K. Yoshida, Minimal surfaces in q-deformed $A d S_{5} \times S^{5}$ string with Poincaré coordinates, arXiv:1410.5544 [INSPIRE].

[46] O. Lunin, R. Roiban and A.A. Tseytlin, Supergravity backgrounds for deformations of $A d S_{n} \times S^{n}$ supercoset string models, Nucl. Phys. B 891 (2015) 106 [arXiv:1411.1066] [INSPIRE].

[47] B. Hoare, Towards a two-parameter q-deformation of $A d S_{3} \times S^{3} \times M^{4}$ superstrings, Nucl. Phys. B 891 (2015) 259 [arXiv:1411.1266] [InSPIRE].

[48] I. Kawaguchi, T. Matsumoto and K. Yoshida, Jordanian deformations of the $A d S_{5} \times S^{5}$ superstring, JHEP 04 (2014) 153 [arXiv:1401.4855] [INSPIRE].

[49] O. Lunin and J.M. Maldacena, Deforming field theories with $\mathrm{U}(1) \times \mathrm{U}(1)$ global symmetry and their gravity duals, JHEP 05 (2005) 033 [hep-th/0502086] [INSPIRE].

[50] S. Frolov, Lax pair for strings in Lunin-Maldacena background, JHEP 05 (2005) 069 [hep-th/0503201] [INSPIRE]. 
[51] T. Matsumoto and K. Yoshida, Lunin-Maldacena backgrounds from the classical Yang-Baxter equation - Towards the gravity/CYBE correspondence, JHEP 06 (2014) 135 [arXiv: 1404.1838] [INSPIRE].

[52] A. Hashimoto and N. Itzhaki, Noncommutative Yang-Mills and the AdS/CFT correspondence, Phys. Lett. B 465 (1999) 142 [hep-th/9907166] [INSPIRE].

[53] J.M. Maldacena and J.G. Russo, Large-N limit of noncommutative gauge theories, JHEP 09 (1999) 025 [hep-th/9908134] [INSPIRE].

[54] D. Dhokarh, S.S. Haque and A. Hashimoto, Melvin twists of global $A d S_{5} \times S^{5}$ and their non-commutative field theory dual, JHEP 08 (2008) 084 [arXiv:0801.3812] [INSPIRE].

[55] T. McLoughlin and I. Swanson, Integrable twists in AdS/CFT, JHEP 08 (2006) 084 [hep-th/0605018] [INSPIRE].

[56] T. Matsumoto and K. Yoshida, Integrability of classical strings dual for noncommutative gauge theories, JHEP 06 (2014) 163 [arXiv: 1404.3657] [INSPIRE].

[57] P. Basu and L.A. Pando Zayas, Chaos rules out integrability of strings in $A d S_{5} \times T^{1,1}$, Phys. Lett. B 700 (2011) 243 [arXiv: 1103.4107] [INSPIRE].

[58] P.M. Crichigno, T. Matsumoto and K. Yoshida, Deformations of $T^{1,1}$ as Yang-Baxter $\sigma$-models, JHEP 12 (2014) 085 [arXiv:1406.2249] [INSPIRE].

[59] A. Catal-Ozer, Lunin-Maldacena deformations with three parameters, JHEP 02 (2006) 026 [hep-th/0512290] [INSPIRE].

[60] T. Matsumoto and K. Yoshida, Integrable deformations of the $A d S_{5} \times S^{5}$ superstring and the classical Yang-Baxter equation - Towards the gravity/CYBE correspondence, J. Phys. Conf. Ser. 563 (2014) 012020 [arXiv:1410.0575] [inSPIRE].

[61] V.E. Hubeny, M. Rangamani and S.F. Ross, Causal structures and holography, JHEP 07 (2005) 037 [hep-th/0504034] [INSPIRE].

[62] I. Kawaguchi, T. Matsumoto and K. Yoshida, A Jordanian deformation of AdS space in type IIB supergravity, JHEP 06 (2014) 146 [arXiv: 1402.6147] [INSPIRE].

[63] C.P. Herzog, M. Rangamani and S.F. Ross, Heating up Galilean holography, JHEP 11 (2008) 080 [arXiv:0807.1099] [InSPIRE].

[64] J. Maldacena, D. Martelli and Y. Tachikawa, Comments on string theory backgrounds with non-relativistic conformal symmetry, JHEP 10 (2008) 072 [arXiv:0807.1100] [INSPIRE].

[65] A. Adams, K. Balasubramanian and J. McGreevy, Hot spacetimes for cold atoms, JHEP 11 (2008) 059 [arXiv:0807.1111] [INSPIRE].

[66] T. Matsumoto and K. Yoshida, Schrödinger geometries from the gravity/CYBE correspondence, to appear.

[67] S. Schäfer-Nameki, M. Yamazaki and K. Yoshida, Coset construction for duals of non-relativistic CFTs, JHEP 05 (2009) 038 [arXiv:0903.4245] [INSPIRE].

[68] M. Sakaguchi and K. Yoshida, Super Schrödinger algebra in AdS/CFT, J. Math. Phys. 49 (2008) 102302 [arXiv:0805.2661] [InSPIRE].

[69] M. Sakaguchi and K. Yoshida, More super Schrödinger algebras from PSU $(2,2 \mid 4)$, JHEP 08 (2008) 049 [arXiv: 0806.3612] [INSPIRE]. 
[70] M. Sakaguchi and K. Yoshida, Supersymmetric extensions of non-relativistic scaling algebras, Symmetry 4 (2012) 517.

[71] S.A. Hartnoll and K. Yoshida, Families of IIB duals for nonrelativistic CFTs, JHEP 12 (2008) 071 [arXiv:0810.0298] [INSPIRE].

[72] L.F. Alday, G. Arutyunov and S. Frolov, Green-Schwarz strings in TsT-transformed backgrounds, JHEP 06 (2006) 018 [hep-th/0512253] [INSPIRE].

[73] T. Buscher, A symmetry of the string background field equations, Phys. Lett. B 184 (1987) 59 [InSPIRE].

[74] T. Buscher, Path-integral derivation of quantum duality in non-linear sigma models, Phys. Lett. B 201 (1988) 466 [INSPIRE].

[75] P. Meessen and T. Ortín, An $\mathrm{SL}(2, Z)$ multiplet of nine-dimensional type-II supergravity theories, Nucl. Phys. B 541 (1999) 195 [hep-th/9806120] [INSPIRE]. 